\title{
BMJ Open Quality Snapshot of current carotid artery stenting practice and accreditation in the USA
}

To cite: Sacks D, Farrell MB, Katzen BT, et al. Snapshot of current carotid artery stenting practice and accreditation in the USA. BMJ Open Quality 2019;8:e000671. doi:10.1136/ bmjoq-2019-000671

- Additional material is published online only. To view please visit the journal online (http://dx.doi.org/10.1136/ bmjoq-2019-000671).

Received 25 February 2019 Revised 16 September 2019 Accepted 21 September 2019

Check for updates

(c) Author(s) (or their employer(s)) 2019. Re-use permitted under CC BY-NC. No commercial re-use. See rights and permissions. Published by BMJ.

'Department of Radiology/ Interventional Radiology, The Reading Hospital and Medical Center, West Reading, Pennsylvania, USA

${ }^{2}$ Research, Intersocietal Accreditation Commission, Ellicott City, Maryland, USA ${ }^{3}$ Miami Cardiac and Vascular Center, Miami, Florida, USA ${ }^{4}$ Intersocietal Accreditation Commission, Ellicott City, Maryland, USA

${ }^{5}$ Department of Surgery, Division of Vascular Surgery, University of Wisconsin School of Medicine and Public Health, Madison, Wisconsin, USA

Correspondence to

Mary Beth Farrell;

farrell@intersocietal.org

David Sacks, ${ }^{1}$ Mary Beth Farrell (D) , ${ }^{2}$ Barry T Katzen, ${ }^{3}$ Mary Lally, Jon S Matsumura, ${ }^{5}$ Nancy Merrill ${ }^{4}$

\section{ABSTRACT}

Objective The aim of this exploratory study was to compare the performance of carotid artery stenting (CAS) best practices between Intersocietal Accreditation Commission (IAC) accredited facilities and non-accredited facilities certified by the Centers for Medicare and Medicaid Services (CMS).

Methods A random, anonymous survey was sent to CMS and IAC accredited facilities querying facility routine performance of 16 CAS procedure components found in published guidelines and utilised during clinical trials.

Results There were 28 responses (response rate $=17 \%$ ). Significant differences were found between the CMS and the IAC facilities for four of 16 procedure measures: determination of modified Rankin Scale score prior to stenting ( $\mathrm{p}=0.012,95 \% \mathrm{Cl} 20 \%$ to $80 \%$ ), accurate measurement of per cent stenosis using electronic callipers ( $p=0.005,95 \% \mathrm{Cl} 24 \%$ to $84 \%$ ), confirmation of anticoagulation with activated clotting time greater than 250 s prior to crossing the lesion $(p=0.03,95 \% \mathrm{Cl} 7 \%$ to $69 \%$ ), and comparison of facility outcomes to accepted benchmarks for stroke and death $(p=0.03,95 \% \mathrm{Cl} 7 \%$ to $69 \%$ ). Overall, IAC facilities performed all 16 procedures more frequently $(97 \%)$ than CMS facilities $(66 \%)(p<0.001$, $95 \% \mathrm{Cl} 24 \%$ to $36 \%$ ).

Conclusions Although the sample size was small, the results demonstrated IAC accredited facilities are more likely to follow best practices, to use quantitative tools to select appropriate patients, and quantitively measure patient-centred clinical outcomes compared with CMS certified facilities. The findings raise the question as to the value of CMS certification versus IAC accreditation as a requirement for reimbursement.

In 2005, the Centers for Medicare and Medicaid Services (CMS) instituted coverage for carotid artery stenting (CAS) procedures for high-risk surgical patients. At that time, there were no recognised multispecialty organisations evaluating CAS procedures. Therefore, CMS established a certification mechanism for evaluating CAS facilities as a condition of reimbursement for CAS procedures. ${ }^{1}$

For initial CMS certification, a facility must meet structural criteria of either having participated in a clinical trial leading to Food and Drug Administration approval of a stenting device or self-attest that it meets minimum standards for equipment, device inventory, staffing and infrastructure. The certification duration is 2 years. To be recertified, facilities submit a log of CAS procedures performed. The log includes patient selection information related to high surgical risk criteria, symptomatic status, modified Rankin Scale (mRS) score if the patient had a history of stroke, per cent diameter stenosis of the artery, and whether there were complications during the hospital stay.

Since the establishment of CMS' certification requirements, stakeholders from professional societies gathered to create standards and develop the Intersocietal Accreditation Commission (IAC) Carotid Artery Stenting accreditation programme based on published guidelines and expert opinion. ${ }^{2}$ The IAC Standards for Carotid Stenting Accreditation define minimum levels of quality based on both processes and outcomes related to the performance of CAS procedures. ${ }^{3}$ The IAC began accrediting CAS facilities in 2011.

The IAC CAS accreditation process requires submission of case logs that include clinical outcomes. IAC staff select a random sample of cases for detailed analysis of the relevant medical records and procedure images. Trained IAC staff also perform a mandatory site visit. The quality of facility operation is determined based on compliance with the Standards. Expert physicians on the IAC CAS Board review the submitted materials and site visit findings to make accreditation decisions. Major deficiencies such as failure to perform neurological assessments must be addressed before accreditation is awarded.

Whether there are differences in adherence to published guidelines and best practices between IAC accredited and CMS non-accredited CAS facilities is not known. ${ }^{2}$ Therefore, this exploratory survey was designed to assess and compare the performance of CAS 
best practices between IAC accredited and non-accredited facilities.

\section{METHODS}

An anonymous survey was conducted in 2017 to compare CAS practices between IAC accredited and non-accredited facilities (online supplementary file A). Non-IAC accredited facilities were randomly selected from a pool of CMS certified facilities. The IAC and CMS facilities selected were mutually exclusive. The same survey was used for both groups, but different survey media was utilised due to differences in the contact information available.

\section{Centers for Medicare and Medicaid Services}

A list of CMS certified facilities was obtained from the CMS.gov website. ${ }^{4}$ At the time the file was retrieved, there were 1366 certified facilities. The data available included facility name, address, provider number, and the effective date of certification.

The facility name and address were verified on the internet for a consecutive sample of 100 facilities to test the integrity of the list. Out of those 100 facilities, one facility was no longer in business. Thus, it was assumed that the information was incorrect for $1 \%$ of the list. Therefore, at least a $10 \%$ random sample plus $1 \%(\mathrm{n}=152)$ was selected from the 1366 CMS certified facilities. The selection was made using a random number generator without replacement. A paper survey was mailed to the selected CMS facilities on at least three separate occasions in January, February and March 2017.

\section{Intersocietal Accreditation Commission}

The same survey was sent electronically to the technical directors of all accredited IAC facilities twice in August 2017; 2 weeks apart.

\section{Survey}

The survey consisted of a combination of dichotomous, checkbox, multiple-choice and free text questions. There were two categories of questions: demographic items (five) and procedure metrics (16). Respondents were asked to indicate if they routinely performed 16 procedure components (table 1). These components were selected from protocols found in the literature, required for CMS certification, utilised during CAS clinical trials, and included in documentation and procedure quality variables assessed in the IAC accreditation process. ${ }^{256}$

For categorical variables, the frequency and percentage were calculated. For continuous variables, the total, median and range were reported. A summed score and

Table 1 Performance of self-reported best practice procedure components

\begin{tabular}{|c|c|c|c|c|c|c|}
\hline \multirow[b]{2}{*}{ Best practice } & \multirow[b]{2}{*}{$\begin{array}{l}\text { CMS (\%) } \\
(n=22)\end{array}$} & \multirow[b]{2}{*}{$\begin{array}{l}\text { IAC (\%) } \\
(n=6)\end{array}$} & \multirow[b]{2}{*}{$\begin{array}{l}\text { Overall } \\
(\%)(n=28)\end{array}$} & \multirow{2}{*}{$\begin{array}{l}\text { Fisher's exact } \\
\text { comparing CMS } \\
\text { versus IAC p value }\end{array}$} & \multicolumn{2}{|c|}{$\begin{array}{l}95 \% \mathrm{Cl} \text { of the } \\
\text { frequencies }\end{array}$} \\
\hline & & & & & $\begin{array}{l}\text { Lower } \\
(\%)\end{array}$ & $\begin{array}{l}\text { Upper } \\
(\%)\end{array}$ \\
\hline mRS pre-stent & 36 & 100 & 50 & 0.01 & 20 & 80 \\
\hline NIHSS pre-stent & 77 & 100 & 82 & 0.55 & -18 & 43 \\
\hline DSA including head and neck pre-stent & 77 & 66 & 75 & 0.62 & -21 & 49 \\
\hline $\begin{array}{l}\text { Measure per cent stenosis using NASCET } \\
\text { criteria }\end{array}$ & 68 & 100 & 75 & 0.29 & -10 & 53 \\
\hline Embolic protection device use & 91 & 100 & 93 & 1.00 & -31 & 28 \\
\hline DSA including head and neck post-stent & 73 & 83 & 75 & 1.00 & -32 & 36 \\
\hline NIHSS 24 hours post-stent & 77 & 100 & 82 & 0.55 & -18 & 43 \\
\hline$\sim 30$-day post-stent neurological assessment & 82 & 100 & 86 & 0.55 & -22 & 39 \\
\hline 30-day post-stent follow-up mRS & 64 & 100 & 71 & 0.14 & -6 & 57 \\
\hline 30-day post-stent follow-up NIHSS & 64 & 100 & 71 & 0.14 & -6 & 57 \\
\hline 30-day post-stent outcomes stroke and death & 73 & 100 & 79 & 0.29 & -14 & 48 \\
\hline 30-day non-invasive study & 59 & 100 & 68 & 0.14 & -2 & 59 \\
\hline Benchmark for stroke and death & 50 & 100 & 61 & 0.03 & 7 & 69 \\
\hline Overall & 66 & 97 & 73 & $<0.001$ & 24 & 36 \\
\hline
\end{tabular}

ACT, activated clotting time; CMS, Centers for Medicare and Medicaid Service; DSA, digital subtracted angiography; IAC, Intersocietal Accreditation Commission; mRS, Modified Rankin Scale; NASCET, North American Symptomatic Carotid Endarterectomy Trial; NIHSS, National Institute of Health Stroke Scale. 
percentage by metric and group were calculated. Comparisons between groups were made using Fisher's exact test. The significance level was set at 0.05 , with the $95 \%$ CI reported. Statistical analyses were performed using IBM SPSS V.22.0.

As the list of CMS certified facilities included facilities that potentially might have participated in the Carotid Revascularization and Medical Management for Asymptomatic Carotid Stenosis Study Trial (CREST-2), a further subanalysis was performed to compare the best practices of CMS facilities that indicated participation in the CREST-2 and those that did not participate in CREST-2. ${ }^{7}$ This evaluation was done for exploratory purpose, and as there was only a small number of facilities, a statistical analysis was not performed. The frequency and percentage were reported for each group and overall.

A typical response rate for email surveys is $2 \%-5 \%$, for generic postal surveys 7.5\%, and for personalised postal surveys $10.5 \%{ }^{8}$ Therefore, a minimum response rate of $10.5 \%$ was established for all surveys due to the mixed media survey methods used.

The research protocol was reviewed by an institutional review board and deemed to be exempt as the survey was voluntary, anonymous, and did not involve the collection of protected health information. An explanation was provided at the beginning of the survey, and all respondents indicated their willingness to participate. As an incentive to participate in the survey, a $\$ 100$ charitable donation was offered.

\section{Patient and public involvement statement}

Patients were not involved in the design or data collection for this study.

\section{RESULTS}

Paper surveys were sent to 152 randomly selected CMS facilities, and electronic surveys of the same questions were sent to 6 IAC facilities. There were 28 responses (overall response rate $=17 \%$ ) to the survey (CMS=22 $(15 \%)$ and $\mathrm{IAC}=6(100 \%))$.

\section{Demographic data}

Most of the responding facilities were medium-sized with between 100 and 500 beds $(n=23,82 \%)$. The rest of the facilities were almost equally distributed between small facilities, less than 100 beds $(n=2,7 \%)$, and large facilities, more than 500 beds $(n=3,11 \%)$. The median number of procedures performed per facility annually was 13 (range 3-90).

The median number of physicians performing CAS per facility was 2 (range 1-8). Most facilities had a combination of physician specialties performing procedures. Of the total reported, vascular surgeons $(n=18,36 \%)$ and interventional cardiologists $(n=13,26 \%)$ were the most frequent followed by interventional radiologists $(n=10$, $20 \%)$. Interventional neurologists $(\mathrm{n}=5,10 \%)$ and neurosurgeons $(n=4,8 \%)$ represented the smallest numbers.
Most facilities participated in at least one registry or clinical study $(n=20,71 \%)$. The American College of Cardiology's National Cardiovascular Data Registry-Peripheral Vascular Intervention (NCDR-PVI ${ }^{\mathrm{TM}}$ ) was the most common registry $(n=10,36 \%)$ followed by the CREST-2 registry $(n=8,29 \%)$. Facilities also participated in the following registries: the Society for Vascular Surgery Vascular Quality Initiative (SVS-VQI) $(\mathrm{n}=6$, 21\%); the Safety and Efficacy Study for Reverse Flow Used During Carotid Artery Stenting Procedure (ROADSTER) ( $\mathrm{n}=2,7 \%)$; and the Carotid Stent Clinical Study for the treatment of carotid artery stenosis in patients at increased risk for adverse events from carotid endarterectomy (SCAFFOLD) $(n=3,11 \%)$. Almost one-third of responding facilities $(n=8,29 \%)$ did not participate in registries. We found that CMS certified facilities were less likely to engage in registries (64\%) compared with IAC facilities $(100 \%)$, although the difference was not significant $(\mathrm{p}=0.14,95 \% \mathrm{CI}-6 \%$ to $57 \%)$.

\section{Procedure metrics overall}

Utilisation of an embolic protection device during the stenting procedure was most frequently utilised $(n=26$, $93 \%)$ by the facilities. Thirty-day post-stenting neurological assessment $(n=24,86 \%), 30$-day mRS score $(n=20$, $71 \%)$, and 30-day National Institute of Health Stroke Scale (NIHSS) score $(n=20,71 \%)$ were also frequently performed (table 1).

The least performed metric was the determination of the degree of stenosis from the catheter angiogram using electronic callipers $(n=13,46 \%)$. Other less frequently performed metrics included mRS assessment prior to stenting $(\mathrm{n}=14,50 \%)$, facility outcomes benchmarking for stroke and death $(\mathrm{n}=17,61 \%)$, and non-invasive diagnostic imaging 30-days post-stent ( $\mathrm{n}=19,68 \%)$.

Overall, the average performance of the 16 procedure metrics by the respondent facilities was $73 \%$.

\section{Procedure metric comparison by accreditation status}

Significant differences were found between the CMS and IAC facilities for four of the 16 procedure measures with the IAC accredited facilities reporting more frequent metric performance (table 1): determination of $\mathrm{mRS}$ score prior to stenting ( $\mathrm{p}=0.012,95 \%$ CI $20 \%$ to $80 \%$ ), accurate measurement of per cent stenosis using electronic callipers $(\mathrm{p}=0.005,95 \%$ CI $24 \%$ to $84 \%$ ), confirmation of anticoagulation with activated clotting time greater than $250 \mathrm{~s}$ prior to crossing the lesion $(\mathrm{p}=0.03$, $95 \%$ CI $7 \%$ to $69 \%$ ), and comparison of facility outcomes to accepted benchmarks for stroke and death $(\mathrm{p}=0.03$, $95 \%$ CI $7 \%$ to $69 \%)$.

Overall, IAC facilities performed all 16 metrics more frequently $(97 \%)$ than CMS facilities $(66 \%) \quad(\mathrm{p}<0.001$, $95 \%$ CI $24 \%$ to $36 \%$ ).

The exploratory analysis evaluating CMS facilities participating in the CREST-2 trial demonstrated that those facilities $(n=3)$ utilised almost all of the 16 best practices when performing CAS with the exception of 
Table 2 Performance of self-reported best practice procedure components for CMS facilities $(n=22)$

\begin{tabular}{|lcc|}
\hline Best practice & $\begin{array}{l}\text { Non-CREST participants (\%) } \\
(\mathbf{n = 1 9 )}\end{array}$ & $\begin{array}{l}\text { CREST participants } \\
\text { (\%) } \\
(\mathbf{n = 3})\end{array}$ \\
\hline mRS pre-stent & 32 & 67 \\
\hline NIHSS pre-stent & 74 & 100 \\
\hline DSA including head and neck pre-stent & 74 & 100 \\
\hline Electronic calliper determination of per cent stenosis & 21 & 100 \\
\hline Measure per cent stenosis using NASCET criteria & 63 & 100 \\
\hline Embolic protection device use & 90 & 100 \\
\hline DSA including head and neck post-stent & 68 & 100 \\
\hline Treated with antiplatelet dual regimen & 79 & 100 \\
\hline ACT >250s & 47 & 100 \\
\hline NIHSS 24 hours post-stent & 74 & 100 \\
\hline 30-day post-stent neurological assessment & 79 & 100 \\
\hline 30-day post-stent follow-up mRS & 58 & 100 \\
\hline 30-day post-stent follow-up NIHSS & 58 & 100 \\
\hline$\sim 30-$ day post-stent outcomes stroke and death & 68 & 100 \\
\hline 30-day non-invasive study & 58 & 67 \\
\hline Benchmark for stroke and death & 42 & 100 \\
\hline Overall & 62 & 100 \\
\hline
\end{tabular}

ACT, activated clotting time; CMS, Centers for Medicare and Medicaid Services; CREST, Carotid Revascularisation and Medical Management for Asymptomatic Carotid Stenosis Study Trial; DSA, digital subtracted angiography; mRS, Modified Rankin Scale; NASCET, North American Symptomatic Carotid Endarterectomy Trial; NIHSS, National Institute of Health Stroke Scale.

two practices (table 2): mRS pre-stent $(67 \%)$ and 30-day non-invasive imaging $(67 \%)$. The omitted best practices for the two variables represented different facilities.

\section{DISCUSSION}

The use of CAS to reduce the risk of stroke and death among patients 70 years or older with carotid artery disease increased significantly from 2007 to $2014 .^{9}$ The American College of Cardiology and American Heart Association guidelines recommend outcomes for periprocedural stroke or mortality of $<6 \%$ for symptomatic patients and $<3 \%$ for asymptomatic patients. ${ }^{10}$ However, favourable results of CAS are highly dependent on the incidence of periprocedural complications. ${ }^{11}$ Periprocedural events are contingent on an operator's skill and experience, patient selection and procedure technique.

Multiple studies have documented the relationship between patient outcomes and competence of the operating team and facility. ${ }^{12-14}$ There is a steep learning curve for CAS with an inverse relationship between operator or facility volume and event rates. Operator and facility volumes were the most significant determinant of periprocedural outcomes. ${ }^{14}$ Recommendations for training criteria and volume requirements vary between professional societies. ${ }^{15}$ The experience and training of physicians performing routine CAS are not captured in the CMS data. ${ }^{13}$
CAS is associated with potential serious complications, and patients should not be subjected to that risk if they are not likely to benefit from the procedure. ${ }^{16}$ In general, symptomatic patients that have $>50 \%$ carotid stenosis and asymptomatic patients with $>80 \%$ carotid stenosis are suitable candidates for CAS. ${ }^{56}$ There is ongoing controversy about indications and ongoing research. However, outside of approved clinical trials, CMS limits reimbursement to symptomatic, high surgical risk patients with stenosis $>70 \%$.

The intent of the CMS certification programme is to confirm that facilities receiving payment for CAS procedures are qualified to perform CAS and achieve acceptable clinical outcomes. ${ }^{7}$ Indeed, the CMS payment memorandum states "facilities and providers that routinely and repeatedly perform this procedure and follow patients for long periods of aftercare have a greater chance of successful outcomes." ${ }^{1}$ However, the current structural focus of the CMS self-certification process does not require facilities to measure outcomes. ${ }^{1}$ A study by Epstein et al found that risk-standardised CAS outcomes fluctuate markedly across the USA; implying that facilities with both high and low adverse events are certified by CMS. $^{17}$

The IAC Standards are based on published guidelines and expert consensus. ${ }^{3}$ They are designed to create best practices with the expectation that these practices will maximise CAS procedure quality and clinical outcomes. 
The Standards also provide metrics that can be used to measure clinical outcomes accurately. The results of this study show that IAC facilities are significantly more compliant with recommended best practices than non-IAC accredited facilities. It is reasonable to assume that this is likely to lead to better clinical outcomes and appropriately selected patients.

One of those best practices is electronic calliper measurement of per cent stenosis. In fact, the CMS memorandum for CAS states that if the degree of stenosis is less than $70 \%$ by angiography at the start of the procedure, CAS should not proceed. However, the memorandum does not suggest a method of ascertaining the degree of stenosis. Per cent stenosis may be determined by visual estimate or measured with electronic callipers. Determination of stenosis by electronic calliper has superior accuracy. Visual estimate of per cent stenosis has been demonstrated to misclassify and overestimate the degree of stenosis, especially in the presence of $50 \%-80 \%$ stenosis. ${ }^{18}$ Overestimation of stenosis may lead to unnecessary interventions. In this study, only $32 \%$ of CMS certified facilities indicated they used electronic callipers to determine per cent stenosis compared with $100 \%$ of IAC accredited facilities.

Assessment of peri-CAS and post-CAS stroke is necessary to determine clinical outcome for a procedure performed to prevent stroke. This requires both a follow-up visit and an objective measure of neurological deficit, such as the NIHSS. While major neurological deficits will be obvious, minor deficits may be overlooked but may still have a large impact on quality of life. ${ }^{19}$ This evaluation is critical in comparing outcomes to national benchmarks.

The findings from this study suggest the utility of accreditation in promoting adherence to best practices of patient selection and assessment of patient outcomes. The comparison of results from CMS facilities to IAC accredited facilities implies that CMS facilities may not necessarily comply with process measures such as the metrics assessed in this study despite CMS requirements. The results indicate it is helpful to have an external entity that audits and provides oversight to ensure best practices such as appropriate patient selection, assessment of clinical outcomes, and comparison of clinical outcomes to nationally accepted benchmarks of patient benefit.

Further, this exploratory examination of a limited number of CMS facilities that also participated in the CREST-2 trial, although notable but not statistically significant, showed they were for the most part very similar to the IAC accredited facilities. This likewise suggests that an external mechanism of accountability, whether in the form of accreditation or participation in a clinical trial, contributes to improving adherence to best practices to ensure quality patient care. Accreditation potentially allows the quality present during a clinical trial to be generalised outside of the clinical trial environment. This is an area for further investigation.

\section{Limitations}

This study is limited by the absence of actual outcomes determination and reporting by non-IAC accredited facilities. Although outcomes data might be available from clinical registries, many of the CMS facilities indicated they did not participate in registries. The study is further limited in that the selected best practices were also not verified directly but self-reported with likely response bias. However, compliance with the selected best practices by IAC facilities was confirmed at the time of the accreditation process site visit. It was assumed that the selected procedure best practices were a determinant of better patient clinical outcomes. It was also assumed that the IAC accreditation process was an accurate assessment of facility quality and best practice adherence.

The study is further limited by the small number of facilities that have sought specialty CAS accreditation by the IAC or the other accreditation organisation; Accreditation for Cardiovascular Excellence (ACE). ACE's accreditation requirements are relatively similar to the IAC's accreditation programme. ${ }^{20}$ The response rate for the survey was low but in line with response rates of other internet surveys. ${ }^{8}$ Finally, inherent selection bias is possible in that the type of individual likely to respond to a survey might also be more likely to adhere to performance guidelines.

\section{CONCLUSIONS}

Our evaluation of guideline adherence and best practices among facilities performing CAS procedures found that despite the small number of facilities, IAC accredited facilities are more likely to follow best practices compared with CMS certified facilities. The most relevant quality measures are appropriate patient selection and reduced risk of postprocedure stroke or death. IAC accredited facilities are more likely than CMS accredited facilities to use quantitative tools to select appropriate patients and quantitatively measure patient-centred clinical outcomes. The results raise the question as to the value of CMS certification as a requirement for reimbursement.

Contributors DS: conception, design, analysis, interpretation, manuscript drafting and revision, and final approval. MBF: conception, design, analysis, interpretation, manuscript drafting and revision, and final approval. BTK: conception, design, interpretation, manuscript revision, and final approval. ML: conception, design, manuscript revision, and final approval. JSM: analysis, interpretation, manuscript drafting and revision, and final approval. NM: conception, design, manuscript revision and final approval.

Funding The Intersocietal Accreditation Commission paid for employees MBF, ML and NM participation. Authors DS, BK and JSM are members of the Intersocietal Accreditation Commission Board of Directors.

Competing interests Authors MBF, ML and NM are employees of the Intersocietal Accreditation Commission. Authors DS, BK and JSM are members of the Intersocietal Accreditation Commission Carotid Stenting Board of Directors.

Patient consent for publication Not required.

Provenance and peer review Not commissioned; externally peer reviewed.

Data availability statement Data are available upon reasonable request. All data relevant to the study are included in the article or uploaded as supplementary information. 
Open access This is an open access article distributed in accordance with the Creative Commons Attribution Non Commercial (CC BY-NC 4.0) license, which permits others to distribute, remix, adapt, build upon this work non-commercially, and license their derivative works on different terms, provided the original work is properly cited, appropriate credit is given, any changes made indicated, and the use is non-commercial. See: http://creativecommons.org/licenses/by-nc/4.0/.

ORCID iD

Mary Beth Farrell http://orcid.org/0000-0001-5958-8791

\section{REFERENCES}

1 U.S. Centers for Medicare \&Medicaid Services. Centers for Medicare and Medicaid services decision memorandum for carotid artery stenting (CAG-00085R). Available: http://www.cms.gov/medicarecoverage-database/details/nca-decision-memo.aspx?NCAld=157 [Accessed 22 Jan 2018].

2 Rosenfield K, Babb JD, Cates CU, et al. SCAI/SVMB/SVS clinical competence statement on carotid stenting: training and credentialing for carotid stenting-multispecialty consensus recommendations: a report of the SCAI/SVMB/SVS writing Committee to develop a clinical competence statement on carotid interventions. J Am Coll Cardiol 2005;45:165-74.

3 The IAC standards for carotid stenting accreditation. Available: http:// www.intersocietal.org/carotid/standards/IACCarotidStentingStanda rds2014.pdf [Accessed 27 Jan 2018].

4 Carotid artery stenting facilities. Available: https://www.cms.gov/ Medicare/Medicare-General-Information/MedicareApprovedFacilitie/ Carotid-Artery-Stenting-Facilities.html [Accessed 22 Jan 2018].

5 Ahn SH, Prince EA, Dubel GJ. Carotid artery stenting: review of technique and update of recent literature. Semin Intervent Radiol 2013;30:288-96.

6 Bates ER, Babb JD, Casey DE, et al. ACCF/SCAI/SVMB/SIR/ASITN 2007 clinical expert consensus document on carotid stenting: a report of the American College of cardiology Foundation Task force on clinical expert consensus documents (ACCF/SCAI/SVMB/SIR/ ASITN clinical expert consensus document Committee on carotid stenting). J Am Coll Cardiol 2007;49:126-70.

7 Locations in the United States and Canada. CREST-2: medical study to prevent stroke caused by plaque buildup in the carotid arteries. Available: http://www.crest2trial.org/locations.html [Accessed 22 Jan 2018].

8 Sinclair M, O'Toole J, Malawaraarachchi M, et al. Comparison of response rates and cost-effectiveness for a community-based survey: postal, internet and telephone modes with generic or personalised recruitment approaches. BMC Med Res Methodol 2012;12:132.
9 Otite FO, Khandelwal P, Malik AM, et al. National patterns of carotid revascularization before and after the carotid revascularization endarterectomy vs stenting trial (crest). JAMA Neurol 2018;75:51.

10 Brott TG, Halperin JL, Abbara S, et al. 2011 ASA/ACCF/AHA/AANN/ AANS/ACR/ASNR/CNS/SAIP/SCAI/SIR/SNIS/SVM/SVS guideline on the management of patients with extracranial carotid and vertebral artery disease: executive summary. A report of the American College of Cardiology Foundation/American Heart Association Task Force on Practice Guidelines, and the American Stroke Association, American Association of Neuroscience Nurses, American Association of Neurological Surgeons, American College of radiology, American Society of Neuroradiology, Congress of Neurological Surgeons, Society of Atherosclerosis Imaging and Prevention, Society for Cardiovascular Angiography and Interventions, Society of Interventional Radiology, Society of Neurolnterventional Surgery, Society for Vascular Medicine, and Society for Vascular Surgery. Circulation 2011;124:489-532.

11 Spacek M, Veselka J. Carotid artery Stenting-Historical context, trends, and innovations. Int J Angiol 2015;24:205-9.

12 Verzini F, Cao P, De Rango P, et al. Appropriateness of learning curve for carotid artery stenting: an analysis of periprocedural complications. J Vasc Surg 2006;44:1205-11.

13 Nallamothu BK, Gurm HS, Ting $\mathrm{HH}$, et al. Operator experience and carotid stenting outcomes in Medicare beneficiaries. JAMA 2011;306:1338-43.

14 Gray WA, Rosenfield KA, Jaff MR, et al. Influence of site and operator characteristics on carotid artery stent outcomes: analysis of the capture 2 (carotid ACCULINK/ACCUNET post approval trial to uncover rare events) clinical study. JACC Cardiovasc Interv 2011;4:235-46.

15 Parlani G, De Rango P, Verzini F, et al. Safety of carotid stenting (Cas) is based on institutional training more than individual experience in large-volume centres. Eur J Vasc Endovasc Surg 2013;45:424-30.

16 Noiphithak R, Liengudom A. Recent update on carotid endarterectomy versus carotid artery stenting. Cerebrovascular Diseases 2017;43:68-75.

17 Epstein AJ, Yang L, Yang F, et al. A comparison of clinical outcomes from carotid artery stenting among US hospitals. Circulation 2014;7:574-80.

18 Rajebi MR, Benenati MJ, Schernthaner MB, et al. Reliability and accuracy of simple visual estimation in assessment of peripheral arterial stenosis. J Vasc Intervent Radiol 2015;26:890-6.

19 Howard VJ, Meschia JF, Lal BK, et al. Carotid revascularization and medical management for asymptomatic carotid stenosis: protocol of the CREST-2 clinical trials. Int J Stroke 2017;12:770-8.

20 ACE Accredited Facilities. Accreditation for cardiovascular excellence. Available: http://www.cvexcel.org/AccreditedFacilities. aspx [Accessed 29 Jan 2018]. 